\title{
Falla de tratamiento en neumonía adquirida en la comunidad: coccidioidomicosis en un viajero
}

\author{
Hernán Cabello, Gonzalo Labarca, Sebastián Fernández-Bussy, Francisca Cabello, \\ Yumay Pires, Rodrigo Soto y Luis Thompson
}

\section{Treatment failure in community-adquired pneumonia: coccidioidomycosis in a traveler patient}

Treatment failure in community-acquired pneumonia is defined as a clinical condition with inadequate response to antimicrobial therapy. Resistant and unusual microorganisms and noninfectious causes are responsible for treatment failure. Coccidioides immitis is a fungus that causes pneumonia in the northern hemisphere, especially in the United States and northern Mexico. We report a case of pulmonary coccidioidomycosis imported from Mexico to Chile. After a comprehensive study, histopathology was able to establish Coccidiodes immitis as the causative agent, achieving clinical and radiological improvement with antifungal therapy.

Key words: Coccidioidomycosis, travel, pneumonia, treatment failure.

Palabras clave: Coccidiodiomicosis, viajero, neumonía, falla de tratamiento.

\section{Introducción}

$\mathrm{L}$ a falla de tratamiento (FT) de la neumonía adquirida en la comunidad (NAC) se define como una respuesta clínica inadecuada en presencia de una terapia antimicrobiana apropiada. Clínicamente se puede traducir en una progresión de la infección, aparición de complicaciones e incluso la muerte ${ }^{1}$. El $40 \%$ de las causas de FT son infecciosas, donde destacan los microorganismos con resistencia a los antimicrobianos habituales (por ej. S. pneumoniae resistente a penicilina). Otras causas de FT de menor frecuencia son microorganismos no cubiertos por la terapia habitual como Staphylococcus aureus, ya sea sensible (SAMS) o resistente a meticilina (SAMR), Pseudomonas aeruginosa, Mycobacterium tuberculosis, Nocardia spp., Acinetobacter spp., Pneumocystis jiroveci y neumonías de etiologías fúngicas como Cryptococcus neoformans, Paracoccidioides brasiliensis, Histoplasma capsulatum, Coccidiodes immitis, entre otras ${ }^{1,2}$.

Se describe el caso de una paciente adulta inmunocompetente que consultó por un cuadro de NAC que no respondió al tratamiento habitual, en quien se observó una progresión clínica-radiológica. Se realizó un estudio etiológico extenso en el cual se logró reconocer mediante patomorfología el diagnóstico de una coccidioidomicosis.

\section{Caso clínico}

Paciente de 50 años de sexo femenino, fumadora de 20 paquetes de cigarrillos al año, de nacionalidad peruana radicada en Chile. Con antecedentes de cáncer cérvico-uterino tratado con cirugía y radio-quimioterapia en remisión clínica, diabetes mellitus insulinorequiriente, dislipidemia y una primoinfección por tuberculosis en la infancia.

Consultó en el Servicio de Urgencia de nuestro centro por un cuadro brusco de $4 \mathrm{~h}$ de evolución, iniciado durante un vuelo en avión, de disnea súbita asociada a dolor urente intenso (EVA 10/10) con tope inspiratorio en región precordial y cara lateral del hemitórax izquierdo. Al ingreso se le describe taquicárdica, eupneica, afebril, con saturación oximétrica de $96 \%$ respirando aire ambiental. En el examen pulmonar destacaban tenues crepitaciones en base del hemitórax izquierdo, sin edema de extremidades ni signos de trombosis venosa profunda. No tenía tos ni expectoración. Se realizaron exámenes de laboratorio: hemograma con 8.200 leucocitos, con 10\% de baciliformes; PCR 1,8 mg/dl (valor normal < $1 \mathrm{mg} / \mathrm{dl}$ ); enzimas cardíacas y gases arteriales normales; dímero-D $800 \mathrm{ng} / \mathrm{ml}$. Una radiografía de tórax mostró consolidación de la língula. Ante la sospecha de un tromboembolismo pulmonar (TEP) se realizó una angio-TAC de tórax que confirmó la imagen de consolidación, descartando un TEP (Figura 1a). Un cintigrama V/Q también resultó negativo para TEP. Un electrocardiograma reflejó una taquicardia sinusal.

Con el diagnóstico de ingreso de NAC se inició tratamiento antimicrobiano empírico con ceftriaxona y moxifloxacina i.v. El estudio etiológico de la neumonía, mediante antígeno urinario para Streptococcus pneumo-
Clínica Alemana-Universidad del Desarrollo, Santiago. Chile. Departamento de Medicina Interna (HC, SFB, RS, LT). Departamento de Anatomía Patológica (YP). Programa de Especialidad en Medicina Interna, Pontificia Universidad Católica, Santiago (GL). Universidad de Valparaíso, Valparaíso, Chile (FC).

Recibido: 9 de abril de 2013 Aceptado: 25 de julio de 2013

Correspondencia a: Hernán Cabello Araya hcabello@alemana.cl 


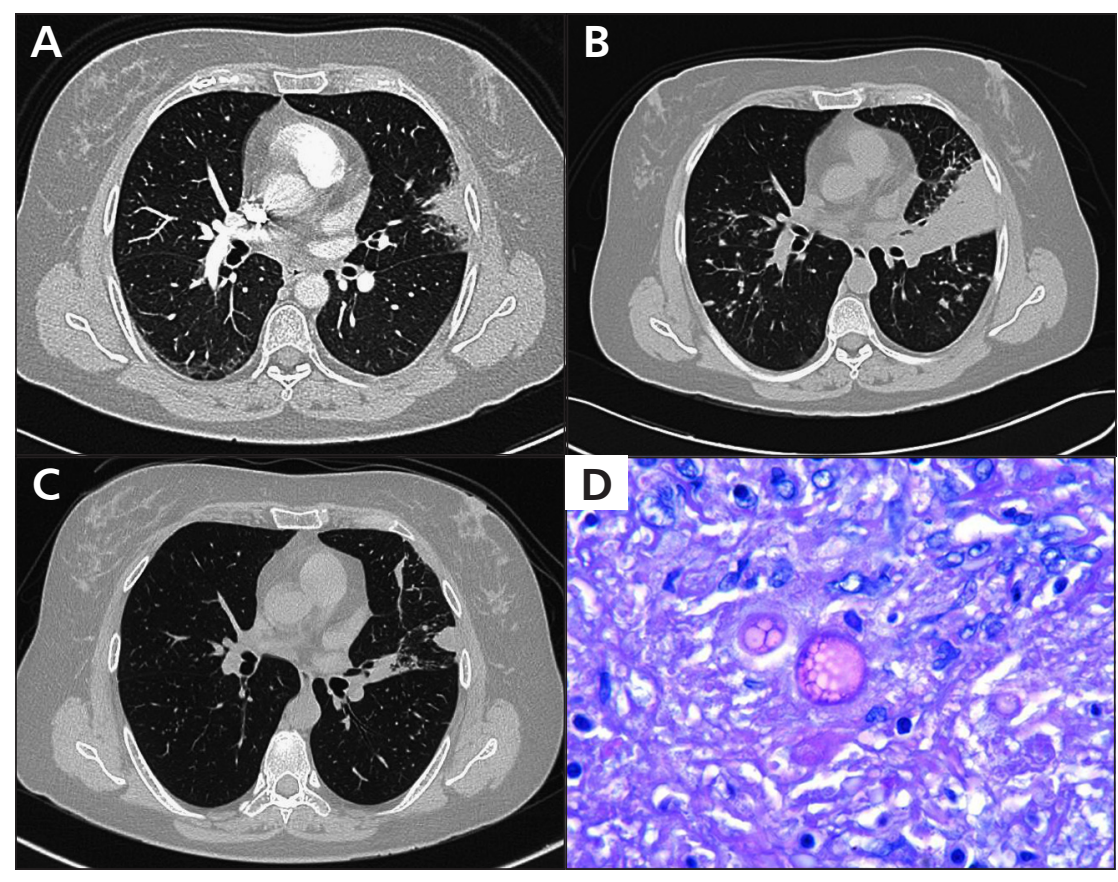

Figura 1. TAC de tórax con los cambios radiográficos. a) TAC inicial con signos de consolidación en língula; b) aumento de tamaño de la condensación lingular con broncograma aéreo asociado a múltiples infiltrados nodulares bilaterales; c) TAC de control en tratamiento con itraconazol, con disminución de la condensación y de los infiltrados pulmonares; d) Tinción de Gomori-Grocott de la muestra de tejido pulmonar obtenida mediante biopsia quirúrgica, se aprecia la presencia de esférulas y endósporas, compatible con coccidioidomicosis.

niae y Legionella pneumophila, IgM para Mycoplasma pneumoniae, hemocultivos y cultivo de secreción bronquial, resultó negativo.

La paciente evolucionó con fiebre desde el segundo día de tratamiento antimicrobiano, con ascenso progresivo de la PCR, con un valor máximo de $17 \mathrm{mg} / \mathrm{dl}$. Clínicamente evolucionó estable y hacia el cuarto día cedió la fiebre y la PCR disminuyó a $12 \mathrm{mg} / \mathrm{dl}$, por lo que se dio de alta a su domicilio con tratamiento antimicrobiano oral con cefuroxima y moxifloxacina hasta completar 14 días.

A las dos semanas del alta, la paciente persistió con fiebre intermitente y apareció una disnea de esfuerzos moderados; presentaba una saturación de oxígeno de 93\% respirando aire ambiental. Entre los exámenes de laboratorio de control destacó una persistencia de la PCR elevada $(12 \mathrm{mg} / \mathrm{dl})$, la velocidad de eritrosedimentación sobre $100 \mathrm{mmHg} 1^{\circ} \mathrm{h}$, procalcitonina normal, el recuento de leucocitos era normal con 5,6\% de eosinófilos. Una nueva radiografía y tomografía axial computada (TAC) de tórax mostraron un aumento del tamaño de la consolidación lingular con broncograma aéreo y aparición de múltiples infiltrados nodulares bilaterales (Figura 1b). La paciente fue reinternada iniciándose tratamiento antimicrobiano empírico con claritromicina y se realizó una fibrobron- coscopia con lavado bronquioalveolar (LBA) y biopsias transbronquiales. La vía aérea se observó de aspecto normal, sin secreciones. Las muestras de biopsias fueron escasas por lo que no permitieron demostrar lesiones significativas. Los cultivos del LBA fueron negativos, así como las bacilocopias y la IF para Pneumocystis jiroveci; tampoco se detectaron elementos micóticos.

La paciente evolucionó los siguientes 10 días con fiebre intermitente y deterioro lento y progresivo del intercambio gaseoso. La imagen radiológica mostró un aumento de tamaño de las lesiones y pérdida del volumen pulmonar.

Se realizó una biopsia pulmonar quirúrgica por videotoracoscopia, la que resultó sin incidentes. Los estudios bacteriológicos en el tejido pulmonar fueron negativos (Gram y cultivo corriente, tinción de Ziehl Neelsen, cultivos de Koch). Una RPC para Mycobacterium spp. también resultó negativa.

El estudio histopatológico pulmonar mostró granulomas intraparenquimatosos y subpleurales no caseificantes, constituídos por histiocitos epitelioídeos, escasos linfocitos y necrosis focal. Hacia la periferia de los granulomas existía infiltrado linfocitario y de granulocitos eosinófilos. Con tinción de Gomori-Grocott se observaron esférulas inmaduras y maduras, estas últimas con endosporas evidentes con tinción de ácido peryódico-de Schiff(PAS). En las tinciones de Gram, Warthin-Starry y Ziehl Neelsen no se observaron bacilos, cocáceas ni bacilos alcohol-ácido resistentes. En dos muestras se observó en la periferia del proceso inflamatorio granulomatoso, tejido conectivo laxo en alvéolos y conductos alveolares. El diagnóstico histológico concluyó una coccidioidomicosis con reacción granulomatosa y patrón de neumonía en organización focal (Figura 1d).

Al reinterrogar la paciente, se rescató el antecedente de un viaje reciente al norte de México, específicamente a la ciudad de Hermosillo (Sonora) y a la frontera de México con E.U.A (Arizona), en donde estuvo cuatro días antes de su regreso vía Perú a Chile. Con este antecedente y la presencia de esférulas con endosporas en la histopatología, se confirmó el diagnóstico de coccidioidomicosis. Se reemplazó el tratamiento antibacteriano por itraconazol $200 \mathrm{mg}$, dos veces al día, por 12 meses, logrando una regresión completa de las lesiones pulmonares y normalización de los parámetros inflamatorios (Figura 1c).

\section{Discusión}

La coccidioidomicosis es una infección fúngica producida por Coccidioides immitis y Coccidioides posadasii. Se encuentra principalmente en climas secos y áridos de ciertas regiones del hemisferio occidental, la gran mayoría dentro de $40^{\circ}$ latitud norte y sur. Demográficamente es una infección característica de regiones como California, Nuevo México, Arizona y Texas. En el sur de Arizona se 
ha comunicado que hasta $29 \%$ de todas las NAC corresponden a coccidioidomicosis ${ }^{3}$.

Sin embargo, la infección por coccidiodomicosis también ha sido descrita en América Central (Guatemala, Honduras, Nicaragua) y América del Sur (especialmente en los valles centrales de Venezuela, Colombia, nordeste de Brasil, noroeste de Paraguay y Argentina) $)^{4,5,6}$. También se han reportado casos fuera de estos sitios, pero con el antecedente de visitas a zonas endémicas de esta infección.

El hongo se encuentra en el ambiente como micelios con hifas septadas, las que se fragmentan en artroconidias, con un diámetro de 2-4 $\mu \mathrm{m}$, que son fácilmente aerosolizadas. Luego de ser inhaladas y alojadas distalmente en el tejido pulmonar cambian de estado, formando esférulas; éstas se dividen internamente y producen múltiples endosporas. La ruptura de una esférula, libera endosporas que se diseminan en el tejido, teniendo capacidad de desarrollar nuevas esférulas ${ }^{7}$.

Cerca de $60 \%$ de los pacientes infectados son asintomáticos o desarrollan síntomas inespecíficos tipo " $f u$-like syndrome", síntomas que son tan leves que por lo general no requieren de atención médica. Aquellos pacientes que desarrollan síntomas y requieren de atención médica, lo hacen entre 7 a 21 días después de la inhalación de las artroconidias, siendo en cerca de $25 \%$ de los casos, la presentación clínica similar a la de una $\mathrm{NAC}^{8}$. Los pacientes asintomáticos y entre $36-38 \%$ de los pacientes sintomáticos, se recuperan $\sin$ requerir tratamiento $\mathrm{y}$ quedan con inmunidad de por vida. Sólo 2 a 4\% de los pacientes desarrollarán una diseminación progresiva de la infección'.

Del punto de vista clínico la coccidioidomicosis se puede clasificar en pulmonar e infección diseminada. Las manifestaciones pulmonares son: neumonía aguda, nódulos, cavidades pulmonares (que pueden causar hemoptisis o neumotórax) y neumonía fibrocavitaria crónica. La infección diseminada se puede clasificar en no meníngea o meníngea. Las infecciones no meníngeas más frecuentes son de piel, abscesos subcutáneos, artritis y osteomielitis. La meningitis por $C$. immitis es una de las principales causas de meningitis eosinofílica en los E.U.A. ${ }^{10}$

Respecto al diagnóstico, no existen métodos de detección rápida. El estudio microbiológico inicial consiste en la detección de esférulas mediante muestras de expectoración utilizando tinciones especiales como la tinción con hidróxido de potasio y la tinción de Papanicolaou; sin embargo, la sensibilidad es baja. En países con alta incidencia de coccidioidomicosis, la serología mediante la detección de IgM es una alternativa utilizada; sin embargo, no existen datos consistentes respecto al rendimiento diagnóstico de estos ensayos ${ }^{11,12}$.

El uso de métodos mínimamente invasores son de utilidad en aquellos casos en los que no se ha confirmado la infección y se requiere de un estudio mediante histología o en los que se requieren muestras para estudio microbiológico y serológico. La broncoscopia con LBA y la biopsia transbronquial es la alternativa más usada, en especial en pacientes inmunocomprometidos con compromiso pulmonar difuso secundario a la infección ${ }^{13,14}$.

Tanto el estudio histológico como el crecimiento de Coccidioides spp. en el medio de cultivo son el "estándar de oro" en esta patología. Clínicamente la infección puede manifestarse como un nódulo pulmonar solitario (NPS) llamado "coccidioma", el que debe ser evaluado conforme a los protocolos de estudio de un NPS. Histológicamente el coccidioma está compuesto por granulomas necrosantes, no caseificantes, con un centro necrótico rodeado de inflamación granulomatosa similar a los encontrados en otras infecciones fúngicas; sin embargo, el hallazgo característico es la visualización directa de esférulas y endoesporas en los tejidos ${ }^{15}$.

Sobre 95\% de las infecciones por Coccidioides spp. no requiere tratamiento específico. La indicación de terapia se recomienda en pacientes inmunocomprometidos o con alguna co-morbilidad. El tratamiento se basa en el uso de azoles (itraconazol o fluconazol), con una duración de tres a seis meses. En situaciones especiales, como en una neumonía aguda difusa o cuadros diseminados en pacientes inmunocomprometidos se recomienda el uso de anfotericina B o dosis mayores de azoles por un período que se puede extender hasta un año. Se recomienda seguir las Guías de tratamiento de Infectious Diseases Society of America (IDSA) ${ }^{16,17}$.

Los azoles han demostrado ser fármacos seguros y efectivos. Con fluconazol responden alrededor de $50 \%$ de los pacientes y con itraconazol, un $63 \%$. Por lo anterior podemos concluir que no todos los pacientes que requieren tratamiento responden y debemos señalar que las recaídas de la coccidioidomicosis no meníngea es de alrededor de $30 \%$, mientras que la recaída de la coccidioidomicosis meníngea es de $75 \%$. Incluso algunos pacientes requieren terapia permanente ${ }^{18}$.

El caso presentado es una coccidioidomicosis en una paciente sudamericana (peruana, radicada en Chile) que cursó una NAC con una mala respuesta clínica y radiológica a tratamientos antimicrobianos habituales, sin un microorganismo aislado. Los medios de cultivos corrientes, especiales y las serologías no fueron suficientes para establecer el diagnóstico definitivo. En este caso, el estudio histológico junto con el antecedente del viaje a una zona endémica fueron claves para el diagnóstico y tratamiento de la infección.

No existen reportes de casos importados de coccidioidomicosis previamente. Alviles-Salas y cols. comunicaron un caso de coccidioidomicosis extrapulmonar correspondiente a un paciente de origen mexicano, sin tener antecedente de viaje o consulta en Chile ${ }^{19}$. Este caso refuerza la importancia de obtener una historia clínica 
completa y considerar la coccidioidomicosis como una alternativa diagnóstica en pacientes con nódulos o masas pulmonares que han viajado a áreas endémicas.

\section{Resumen}

La neumonía de evolución tórpida son aquellas en que no se logra una respuesta clínica adecuada con el uso de terapia antimicrobiana. Existen múltiples causas a esta falta de respuesta: resistencia antimicrobiana, microorga- nismos no cubiertos o infecciones por microorganismos atípicos. Coccidioides immitis es un hongo causante de neumonía en el hemisferio norte, especialmente en E.U.A y norte de México. No existen reportes de casos pulmonares importados en Chile. Presentamos el caso de una mujer adulta con una neumonía que no respondió al tratamiento antimicrobiano habitual. Una vez realizado un estudio exhaustivo, se logró establecer mediante el estudio histopatológico la existencia de una coccidiodomicosis como entidad causal, logrando una respuesta clínica y radiológica favorable al tratamiento antifúngico.

\section{Referencias bibliográficas}

1.- Menéndez R, Torres A. Treatment failure in community-acquired pneumonia. Chest 2007; 132: 1348-55.

2.- Menéndez R, Perpiñá M, Torres A. Evaluation of nonresolving and progressive pneumonia. Semin Respir Infect 2003; 18: 103-11.

3.- Valdivia L, Nix D, Wright M, Lindberg E, Fagan T, Lieberman D, et al.

Coccidioidomycosis as a common cause of community-acquired pneumonia. Emerg Infect Dis 2006; 12: 958-62.

4.- Wang Z Y, Wen S L, Ying K J. A case study of imported pulmonary coccidioidomycosis J Zhejiang Univ Sci B 2011; 12: 298-302.

5.- Crum N F, Lederman E R, Stafford C M, Parrish J S, Wallace M R. Coccidioidomycosis: a descriptive survey of a reemerging disease. Clinical characteristics and current controversies. Medicine (Baltimore) 2004; 83: 149-75.

6.- Negroni R, Arechavala A, Maiolo E. Coccidioidomicosis. Med Cutan Iber Lat Am 2010; 38: 179-88
7.- Center for Diseases Control and Prevention. Coccidioidomycosis (Valley Fever). Disponible en: http://www.cdc.gov/fungal/ coccidioidomycosis/ (Accedido: 21 de febrero de 2013).

8.- Parish J M, Blair J E. Coccidioidomycosis. Mayo Clin Proc 2008; 83: 343-48.

9.- Galgiani J. Coccidioides species. En Mandell GL, Bennett JE, Dolin R, eds. Mandell, Douglas and Bennett's Principles and Practice of Infectious Diseases. 7th Edition, Philadelphia: Churchill Livingstone, Elsevier 2010; p: 3333-44.

10.- Lo Re V 3rd, Gluckman SJ. Eosinophilic meningitis. Am J Med 2003; 114: 217-23.

11.- Ampel NM. New perspectives on coccidioidomycosis. Proc Am Thorac Soc 2010; 7: 181-5.

12.- Kuberski T, Herrig J, Pappagianis D. False-positive IgM serology in coccidioidomycosis. J Clin Microbiol 2010; 48: 2047-9.

13.- Khan R, Sharma O P. Bronchial lavage in tropical pneumonias. Curr Opin Pulm Med 2007; 13: 225-9.
14.- Hage C A, Knox K S, Davis T E, Wheat L J. Antigen detection in bronchoalveolar lavage fluid for diagnosis of fungal pneumonia. Curr Opin Pulm Med 2011; 17: 167-71.

15.- Mukhopadhyay S. Role of histology in the diagnosis of infectious causes of granulomatous lung disease. Curr Opin Pulm Med 2011; 17 : 189-96.

16.- Galgiani J N, Ampel N M, Blair J E, Catanzaro A, Johnson R H, Stevens D A, et al. Coccidioidomycosis. Clin Infect Dis 2005; 41: 1217-23.

17.- Pappas P G. The role of azoles in the treatment of invasive mycoses: review of the Infectious Diseases Society of America guidelines. Curr Opin Infect Dis 2011; 24 (S2): S1-S13.

18.- Valley Fever Center for Excellence. Coccidioidomycosis. Disponible en: http:www.vfce.arizona.edu. Accedido: 6 de abril de 2013.

19.- Aviles-Salas A, Quintero-Cuadra Y, Cornejo-Juárez P. Coccidioidomicosis extrapulmonar. Presentación de un caso y revisión de la literatura. Rev Chilena Infectol 2007; 24: 398-401. 